\title{
Enzyme Immunoassay Using a Reusable Extended-gate Field-Effect-Transistor Sensor with a Ferrocenylalkanethiol- modified Gold Electrode
}

\author{
Masao KaMahori, ${ }^{* \dagger}$ Yu Ishige, ${ }^{*}$ and Maki ShIMOdA** \\ *Central Research Laboratory, Hitachi, Ltd., 1-280 Higashi-Koigakubo, Kokubunji, Tokyo 185-8601, Japan \\ **Hitachi ULSI Systems Co., Ltd., 1-280 Higashi-Koigakubo, Kokubunji, Tokyo 185-8601, Japan
}

\begin{abstract}
A reusable extended-gate field-effect transistor (FET) sensor with an 11-ferrocenyl-1-undecanethiol (11-FUT) modified gold electrode was developed for applying to enzyme immunoassay. It was found that the 11-FUT modified FET sensor detected a thiol compound 50 times or more repeatedly after a treatment with a 5\% hydrogen peroxide solution. The gatevoltage shift of the FET sensor showed a fairly good linearity $\left(R^{2}=0.998\right)$ within a range from $10^{-2}$ to $10^{-6} \mathrm{M}$ on the concentration of 6-hydroxyl-1-hexanethiol, which is a thiol compound, at a Nernstian response of $58.5 \mathrm{mV} / \mathrm{decade}$. The FET-based immunoassay was constructed by combining the 11-FUT modified-FET sensor with the enzyme-linked immunosorbent assay (ELISA), in which the enzyme chemistry of acetylcholinesterase (AChE) was used to generate a thiol compound. The 11-FUT modified FET sensor with an AC voltage at $1 \mathrm{MHz}$ superimposed onto the reference electrode detected the AChE-catalyzed product corresponding to a serum concentration of interleukin $1 \beta$ from 10 to 5000 $\mathrm{pg} / \mathrm{mL}$. In addition, all measurements were successfully performed by using the same FET-sensor chip after a treatment with a $5 \%$ hydrogen peroxide solution.
\end{abstract}

(Received April 23, 2008; Accepted July 28, 2008; Published September 10, 2008)

\section{Introduction}

Enzyme immunoassays (EIAs) are widely used to detect analytes of interest in molecular biology, clinical testing, and environmental applications. The most common detection methods for EIAs are based on spectrophotometric detections, such as those of chemiluminescence and absorbance. These detection methods require the use of a spectrometer for measuring the enzyme-catalyzed chemiluminescence or absorbance of the enzyme-catalyzed products, requiring largescale and expensive instruments. Recently, EIAs based on electrochemical detection have been developed, and are becoming increasingly important. ${ }^{1,2}$ Many research groups have focused on the electrochemical immunosensor, which is based on an enzyme-linked immunosorbent assay (ELISA) system and electrochemical detection. The advantage of electrochemical detection over spectrophotometric detection is the use of inexpensive instrumentation without an optics system, meaning that the system can be easily miniaturized. Nevertheless, the problem still remaining in regard to electrochemical detection is noise due to the background current of the working electrode. Moreover, reducing the sample volume reduces the current produced on the electrode, resulting in lower sensitivity. To enhance the signal intensity of electrochemical detection, enzymatic signal amplification and electrochemical signal amplification methods have been developed. Enzymatic signal amplification was achieved by using alkaline phosphatase as an enzyme labeling, which catalyzes $p$-aminophenolester to

$\dagger$ To whom correspondence should be addressed.

E-mail: masao.kamahori.qj@hitachi.com $p$-aminophenol. ${ }^{1,3}$ On the other hand, a signal amplification with no enzyme was achieved by using a catalytic reaction of $p$-nitrophenol by a gold nanocatalyst. ${ }^{4}$ Both amplification methods used a cycling reaction to accumulate the reaction products. Combining the enzymatic-amplification reaction and the electrochemical-redox-cycling reaction obtained much higher sensitivity. ${ }^{5}$

EIAs based on the potentiometric detection can detect an interfacial potential change induced by biological interactions on a solid surface. One approach was direct immunosensing by using an ion-sensitive field-effect transistor (ISFET) sensor. ${ }^{6}$ The gate of the ISFET sensor is modified with an antibody or an antigen. When an antibody-antigen complex is formed on the gate surface, a surface-charge change is produced. The ISFET sensor can detect a surface-charge change as a shift of the drain current. Even though many research groups have tried to realize this approach, the obtained results have been limited. ${ }^{7}$ The reason for this is that the direct detection of an immunological reaction requires ideal conditions; namely, the size of an electric field produced by charged proteins must be within the Debye length of a sample solution. ${ }^{8}$ When an antibody-antigen complex is shielded by counter ions at distances greater than the Debye length from the gate surface, it cannot be detected by the FET sensor. To solve this key problem, the ion-step method for ISFET-based detection of lysozymes was introduced by Schasfoort et al. ${ }^{9}$ and was further investigated by other research groups. ${ }^{10-12}$ The ion-step method is based on a dynamic measurement method in which an ISFET sensor detects the ionstep response of the release or uptake of protons by protein molecules bound onto the sensor. Additionally, indirect potentiometric detection using an ISFET sensor was combined with ELISA. ${ }^{13-15}$ More recently, a novel enzyme immunoassay 


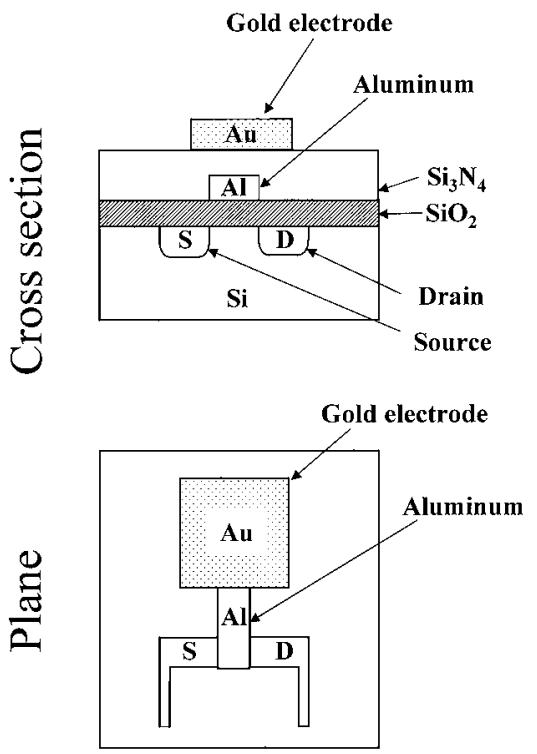

Fig. 1 Cross section and plane structure of extended-gate FET sensor. The gold electrode is connected to the FET with aluminum wire.

based on a potentiometric measurement of molecular adsorption events on a gold electrode of an extended-gate FET sensor has been reported. ${ }^{16}$ As a labeled enzyme, acetylcholinesterase $(\mathrm{AChE})$ is a high-turnover enzyme, and has played the role of generating thiol compounds, which adsorb easily on the gold electrode. The interface potential on the gold electrode of the FET sensor has been shown to shift in the negative direction as a result of the adsorption of a thiol compound on the gold electrode, and the adsorption rate was related to the concentration of the antibody-antigen complex, i.e., the target in the sample. The use of both $\mathrm{AChE}$ and two series of specific monoclonal antibodies resulted in a highly sensitive detection of interleukin $1 \beta$ (IL-1 $\beta$ ), i.e., as low as $1 \mathrm{pg} / \mathrm{ml}$. Since the sensor cannot be reused without any special treatment to remove the adsorbed molecules, the FET-sensor tip as well as the reagents must be disposed of.

In this paper, we report on a reusable extended-gate FET sensor with an 11-ferrocenyl-1-undecanethiol (11-FUT) immobilized gold electrode and its application to an enzyme immunoassay. The enzyme immunoassay system using the 11-FUT modified FET sensor was combined with the enzyme chemistry of AChE and ELISA techniques. The 11-FUT modified FET sensor detected the products of an AChEcatalyzed reaction as a shift in the gate voltage of the FET sensor. In addition, the modified gold electrode of the FET sensor could be used repeatedly after a treatment with a 5\% hydrogen peroxide solution. It was concluded from these results that the 11-FUT modified FET sensor combined with the ELISA system could be used repeatedly to successfully detect IL- $1 \beta$ at concentrations as low as $10 \mathrm{pg} / \mathrm{mL}$.

\section{Experimental}

\section{Chemicals and reagents}

The following chemicals and reagents were used in the experiments: sodium chloride, sodium sulfate anhydrous, sodium dihydrogenphosphate dihydrate, disodium hydrogenphosphate, ethylenediaminetetraacetic acid disodium salt dehydrate, 2-(acetylthio) ethyl trimethylammonium chloride, albumin from

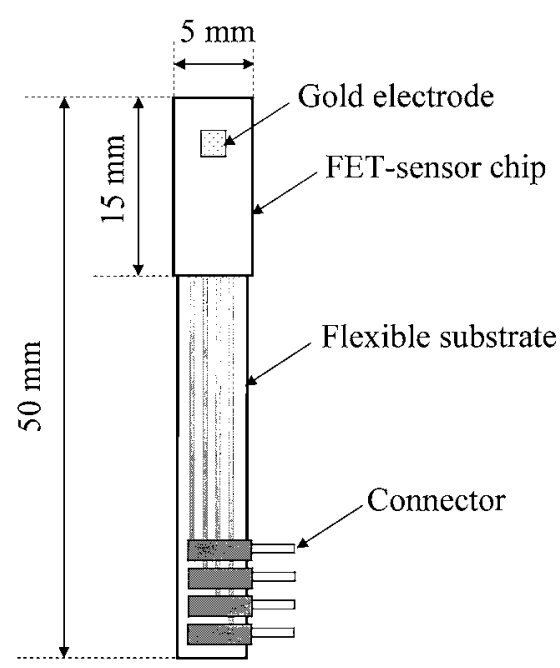

Fig. 2 Schematic view of the extended-gate FET sensor. The FETsensor chip was assembled with a flexible substrate and connected to a 4-pin connector.

bovine serum (BSA), nitric acid, hydrogen peroxide, and ethanol (99.5\%), from Wako Pure Chemical Industries Ltd. (Osaka, Japan); 6-hydroxyl-1-hexanethiol (6-HHT) and 11-ferrocenyl-1undecanethiol (11-FUT) from Dojindo Laboratories (Kumamoto, Japan); and an IL-1 $\beta$ EIA kit, IL-1 $\beta$ (human), acetylcholinesterase-Fab' EIA conjugate, and human plasma from Cayman Chemical (MI, USA).

All reagent solutions were prepared with deionized water (resistivity of $18.2 \mathrm{M} \Omega \mathrm{cm}^{-1}$ or more) from an ultra-pure water system, Model WD500, Yamato Scientific Co. Ltd. (Tokyo, Japan).

Fabrication extended-gate FET sensor and modification of a gold electrode

A schematic view of assembled extended-gate FET sensor chip used in this study is shown in Fig. 1. The FET-sensor chip was assembled with a flexible substrate and connected to a 4-pin connector (Fig. 2). The FET sensor was fabricated using a standard p-well silicon technology. The fundamental characteristics of the FET sensor were described previously. ${ }^{17}$ The FET has an n-channel depletion-type structure formed by $\mathrm{As}^{+}$ion implantation at $120 \mathrm{keV}$ at a dose of $6.0 \times 10^{11}$ molecules $/ \mathrm{cm}^{2}$. The channel region is $2400 \times 5 \mu \mathrm{m}(\mathrm{W} \times \mathrm{L})$, and the gate insulator of the FET consists of a 17.5-nm-thick $\mathrm{SiO}_{2}$ layer formed by wet oxidation under a $1.3-\mu$ m-thick $\mathrm{Si}_{3} \mathrm{~N}_{4}$ layer formed by chemical vapor deposition. The gold electrode $(400 \times 400 \mu \mathrm{m})$ was fabricated by sputter deposition of an adhesion layer of nickel-tungsten alloy, and then a gold layer (100 nm, 99.99\% purity) on the sensing area of the FET. The gold electrode is connected with its gate terminal by an aluminum wire.

An 11-FUT immobilized FET sensor was prepared as follows. The FET-sensor chip was washed with $1 \mathrm{M}$ nitric acid and rinsed twice with deionized water to clean it. The chip was then dipped in a 1-mM 11-FUT ethanol-solution for $1 \mathrm{~h}$. After immobilization, the chip was immersed in a $0.1-\mathrm{M}$ sodium sulfate solution at $90^{\circ} \mathrm{C}$ for $10 \mathrm{~min}$, and rinsed twice with deionized water to clean it. This chip was then dipped in a $0.1-$ M sodium sulfate solution until use. 


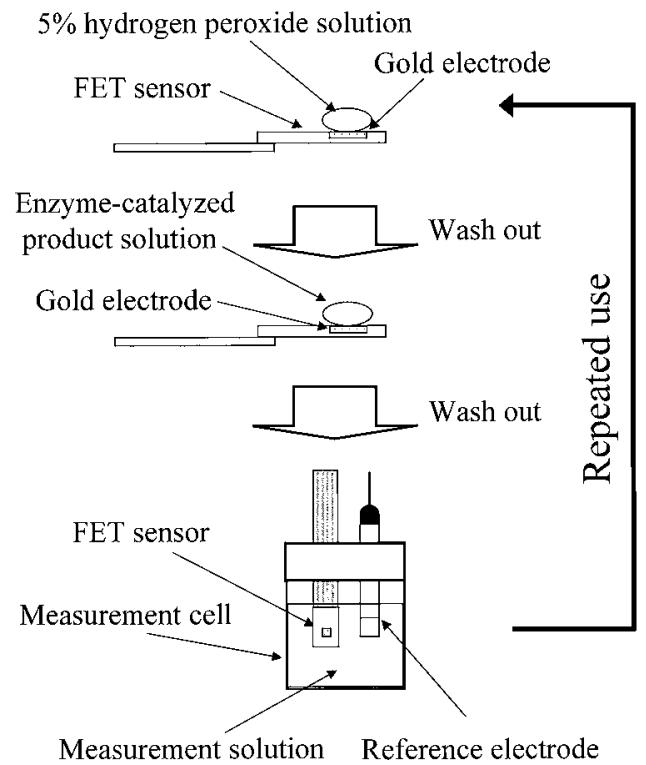

Fig. 3 Scheme of the measurement procedures of thiol compounds in an enzyme-catalyzed product solution using an 11-FUT modified FET sensor.

\section{Enzyme immunoassay}

An enzyme immunoassay based on using monoclonal anti-IL$1 \beta$ labeled with $\mathrm{AChE}$ was conducted by modifying a previous report. ${ }^{16}$ The procedure for the enzyme immunoassay is as follows. Each well of the microtiter plate was coated with a monoclonal antibody for IL- $1 \beta$ (IL- $1 \beta$ capture antibody). First, $100 \mu \mathrm{l}$ of each IL- $1 \beta$ solution at concentrations of $0,10,100$, 1000 , and $5000 \mathrm{pg} / \mathrm{mL}$ in human plasma and $100 \mu \mathrm{l}$ of a covalent conjugate of monoclonal anti-IL-1 $\beta$ Fab' and AChE (AChE: Fab' conjugate) in $0.1-\mathrm{M}$ phosphate buffer $(0.1 \%$ BSA, $0.4-\mathrm{M} \mathrm{NaCl}$ and 1-mM EDTA, pH 7.4) were sequentially added to each well of the microtiter plate, and incubated overnight at $4^{\circ} \mathrm{C}$. Each well was rinsed five times with a washing buffer (0.1-M phosphate buffer, $0.1 \%$ BSA, 0.4-M NaCl, 1-mM EDTA, and 0.05\% Tween $20, \mathrm{pH}$ 7.4) for $\mathrm{B} / \mathrm{F}$ separation. One hundred microliters of 2.5 $\mathrm{mM}$ acetylthiocholine in a $0.1-\mathrm{M}$ phosphate buffer $(0.15-\mathrm{M}$ $\mathrm{NaCl}$ and 1-mM EDTA, $\mathrm{pH}$ 7.4) were added to each well and incubated for $30 \mathrm{~min}$ at room temperature. The hydrolysis of acetylthiocholine by $\mathrm{AChE}$ produces thiocholine as follows:

$$
\begin{array}{r}
\mathrm{CH}_{3} \mathrm{COSCH}_{2} \mathrm{CH}_{2} \mathrm{~N}\left(\mathrm{CH}_{3}\right)_{3} \stackrel{\text { Acetylcholinesterase }}{\longrightarrow} \\
\mathrm{CH}_{3} \mathrm{COOH}+\left(\mathrm{CH}_{3}\right)_{3} \mathrm{NCH}_{2} \mathrm{CH}_{2} \mathrm{SH}
\end{array}
$$

Ten microliters of the reaction-product solution were used for measurements using the extended-gate FET sensor.

\section{Measurement procedures by an extended-gate FET sensor}

The scheme of the measurement procedures of thiol compounds in an enzyme-catalyzed product solution using the 11-FUT modified FET sensor is shown in Fig. 3. First, 11-FUT molecules modified on the FET senor were oxidized by a $5 \%$ hydrogen peroxide solution into the oxidation state. Next, an enzymecatalyzed product solution was put on the 11-FUT modified gold electrode. After the redox reaction between thiol compounds in the enzyme-catalyzed product solution and the 11-FUT molecules, the gold electrode was wash out. Then, the FET sensor and the reference electrode were put into a measurement cell filled with a measurement solution. After a measurement of the drain-current change of the FET sensor, the modified gold electrode of the FET sensor was wash out, and was then oxidized by a $5 \%$ hydrogen peroxide solution. Repeated use of the FET sensor can be performed by repeating a series of operations.

To investigate the dependency of the potential change on the concentration of the thiol compound, a 6-HHT molecule having a $\mathrm{SH}$ residue was used as a reducing reagent. 6-HHT solutions at final concentrations from $10^{-6}$ to $10^{-2} \mathrm{M}$ were used. This molecule was used because $\mathrm{AChE}$-catalyzed products from the enzyme immunoassay contain $\mathrm{SH}$ residues that react to 11-FUT immobilized on the gold surface through a reduction reaction in the same way as 6-HHT. First, the 11-FUT immobilized gold electrode of the FET sensor was dipped in a 5\% hydrogen peroxide solution for $3 \mathrm{~min}$. The 11-FUT molecules were oxidized by the hydrogen peroxide into the oxidation state. Next, $10 \mu \mathrm{L}$ of the 6-HHT solution at each concentration was put on the modified gold electrode, and the reduction reaction was performed for $5 \mathrm{~min}$. After the reaction, the gold electrode was rinsed twice with the measurement solution (0.1-M sodiumsulfate solution). The FET sensor and the reference electrode (RE-1B, BAS, Tokyo, Japan) were then immersed in a measurement cell filled with $3.8 \mathrm{~mL}$ of the measurement solution. The measurement of the drain-current change of the FET sensor was performed by a semiconductor parameter analyzer (Agilent 4155C, Tokyo, Japan) when a 0.1-V DC voltage with a superimposed sine wave $(1 \mathrm{MHz}, 0.2 \mathrm{Vp}$-p) onto the $\mathrm{Ag} / \mathrm{AgCl}$ reference electrode was applied by a function generator (1940 Multifunction Synthesizer, NF Circuit Design Block, Kanagawa, Japan). The amount of the drain-current shift $\left(I_{\mathrm{D}}\right)$ based on the reduction of 11-FUT molecules on the gold electrode was converted into a change of the interface potential by using a gate bias- $I_{\mathrm{D}}$ curve, as reported elsewhere. ${ }^{17}$ After the measurement was finished, the modified gold electrode of the FET sensor was again dipped in a 5\% hydrogen peroxide solution for $3 \mathrm{~min}$. Next, the reaction of the 11-FUT with the 6HHT molecules and the measurement of the FET sensor were performed repeatedly. All measurements were performed by using the same FET-sensor chip.

The measurement procedure of the enzyme immunoassay was as follows. The product of the AChE-catalyzed reaction was measured according to the same procedure as that for the 6HHT molecules. First, the 11-FUT immobilized gold electrode of the FET sensor was dipped into a $5 \%$ hydrogen peroxide solution for $3 \mathrm{~min}$. Ten microliters of the product solution of the AChE-catalyzed reaction were placed on the modified gold electrode. After the redox reaction for $5 \mathrm{~min}$, the gold electrode was rinsed twice with the measurement solution. The FET sensor and the reference electrode (RE-1B, BAS, Japan) were immersed in a measurement cell filled with $3.8 \mathrm{~mL}$ of the measurement solution. The drain-current change of the FET sensor was measured by a semiconductor parameter analyzer (Agilent 4155C, Japan) when a $0.1-\mathrm{V}$ DC voltage with a superimposed sine wave $\left(1 \mathrm{MHz}, 0.2 \mathrm{~V}_{\mathrm{p}-\mathrm{p}}\right)$ onto the $\mathrm{Ag} / \mathrm{AgCl}$ reference electrode was applied by a function generator (1940 Multifunction Synthesizer, NF Circuit Design Block, Japan). After the measurement was finished, initializing with a $5 \%$ hydrogen peroxide solution, a reaction with AChE-catalyzed product, and an electric measurement of the FET sensor were performed repeatedly. All measurements were performed using the same FET-sensor chip.

\section{Results and Discussion}

Fundamental characteristics of 11-FUT modified FET sensor Since the concept of direct immunosensing by using ISFETs, 


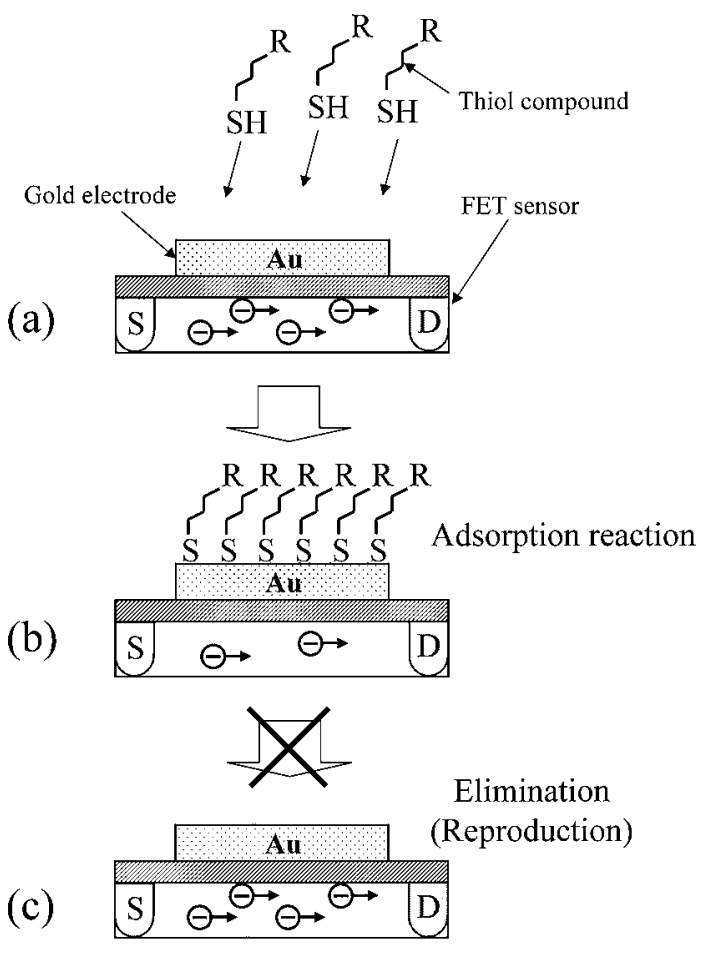

Fig. 4 Principle of the detection of molecular adsorption events by an extended-gate FET sensor. (a) Extended-gate FET sensor with a bare gold electrode before molecular adsorption reaction. (b) Extended-gate FET sensor with the thiol-compound adsorbed gold electrode after a molecular adsorption reaction. (c) Extended-gate FET sensor with a bare gold electrode after removing the adsorbed molecules.

the so-called immuno-FET, was introduced by Schenck, ${ }^{6}$ many types of ISFET-based biosensors have been developed. However, the results obtained by immuno-FET have been limited because of the key problem of the Debye length. ${ }^{8}$ Direct immunosensing requires an ideal condition where an electric field produced by charged molecules is within the Debye length of the sample solution. Also, an indirect immunosensing based on a $\mathrm{pH}$-sensitive FET combined with an ELISA system was developed. ${ }^{13,14}$ Recently, we developed a novel enzyme immunoassay based on a potentiometric measurement of molecular adsorption events by an extended-gate FET sensor. ${ }^{16}$ An enzyme immunoassay by using the extended-gate FET sensor was combined with ELISA, in which the enzyme chemistry of AChE to generate a thiol compound was used. The generating thiol compounds adsorb easily on the gold electrode shown in Figs. 4(a) and (b). The interface potential on the gold electrode of the FET sensor has been shown to shift in the negative direction as a result of the adsorption of a thiol compound on the gold electrode. Since the adsorption rate was related to the concentration of the labeled enzyme, the target in the sample can be estimated from it. However, it was difficult to reuse the FET-sensor tip because of a difficulty of removing the adsorbed molecules as shown in Fig. 4(c).

To solve the above-mentioned problem, we proposed a reusable extended-gate FET sensor with an 11-FUT immobilized gold electrode. Ferrocene is an organometallic compound with the formula $\mathrm{Fe}\left(\mathrm{C}_{5} \mathrm{H}_{5}\right)_{2}$, which is well-known as a sandwich compound. Ferrocene undergoes one-electron oxidation at a low potential, around $0.5 \mathrm{~V} v s$. a saturated calmel electrode, and is often used as an internal standard for calibrating redox (a)

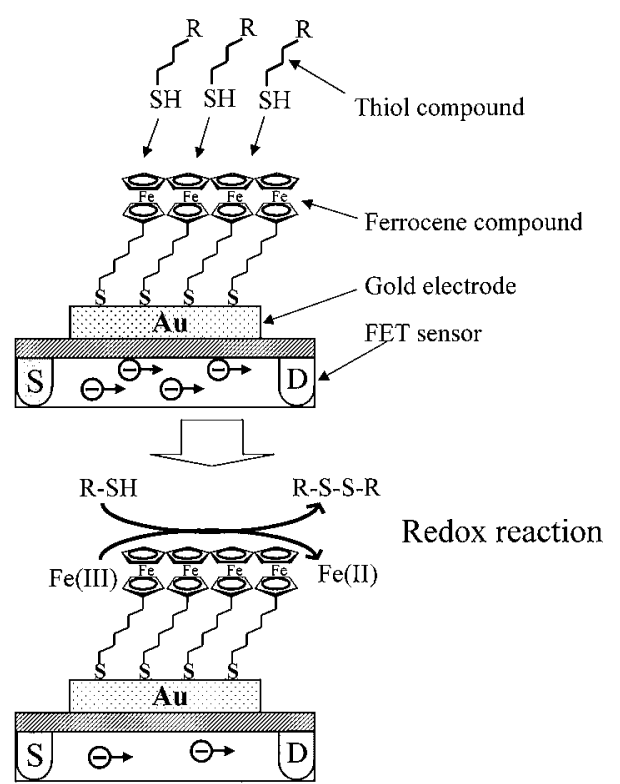

(b)

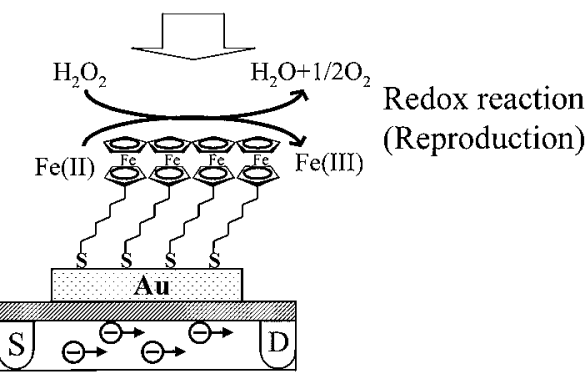

Fig. 5 Principle of detection of the redox reaction on an 11-FUT modified gold electrode by using an extended-gate FET sensor. (a) Extended-gate FET sensor with an 11-FUT modified gold electrode before a redox reaction by the thiol compound. (b) Extended-gate FET sensor with an 11-FUT modified gold electrode after a redox reaction by the thiol compound. (c) Extended-gate FET sensor with an 11-FUT modified gold electrode after a redox reaction by a $5 \%$ hydrogen peroxide solution.

potentials in electrochemistry. Since 11-FUT having an alkanethiol group is a derivative of ferrocene, 11-FUT also shows a very stable redox reaction. Immobilized 11-FUT molecules on the gold electrode can be expected to react with a thiol compound, which is a reducing reagent, as shown in Figs. 5(a) and (b). By the redox reaction, the 11-FUT molecules in oxidation state are converted in the reduction state, which results in an interfacial potential change. It was found that the 11-FUT modified FET sensor could detect this interfacial potential change as a change in the drain-current of the FET sensor. In addition, the 11-FUT molecules in the reduction state can be easily oxidized by a $5 \%$ hydrogen peroxide solution into the oxidation state as shown in Fig. 5(c). Thus, the 11-FUT modified gold electrode of the FET sensor can be used repeatedly after a treatment with a 5\% hydrogen peroxide solution.

The oxidation reaction of the thiol compound in the solution and the reduction reaction of the ferrocene compound immobilized on the surface are given, respectively, as

$$
\begin{aligned}
& (\mathrm{R}-\mathrm{SH})_{\text {Liquid }} \rightleftharpoons \mathrm{e}^{-}+\frac{1}{2}(\mathrm{R}-\mathrm{S}-\mathrm{S}-\mathrm{R})_{\text {Liquid }}, \\
& {[\mathrm{Fc}(\mathrm{III})]_{\text {Surface }}+\mathrm{e}^{-} \rightleftharpoons[\mathrm{Fc}(\mathrm{II})]_{\text {Surface }},}
\end{aligned}
$$




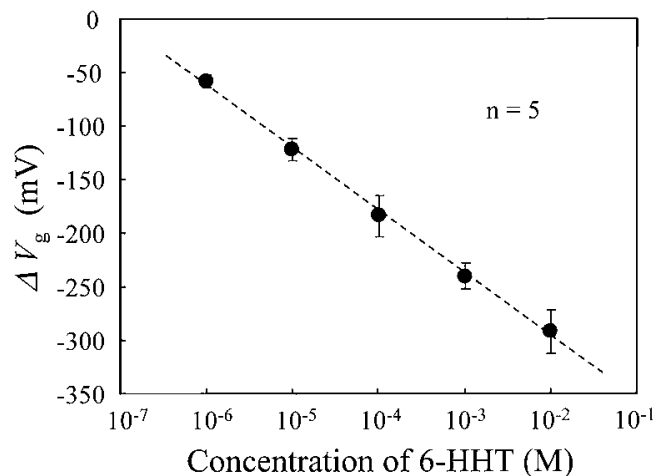

Fig. 6 Concentration of a 6-HHT solution versus gate-voltage change measured by the 11-FUT modified FET sensor.

where $(\mathrm{R}-\mathrm{SH})_{\text {Liquid }}$ is the concentration of the thiol compound in solution (reduction state), $(\mathrm{R}-\mathrm{S}-\mathrm{S}-\mathrm{R})_{\text {Liquid }}$ is the concentration of the thiol compound in solution (oxidation state), [FC(III) $]_{\text {Surface }}$ is the apparent concentration of the ferrocene compound immobilized on the surface (oxidation state), and [FC(II) $]_{\text {Surface }}$ is the apparent concentration of the ferrocene compound immobilized on the surface (reduction state).

The interfacial potential on the surface $\left(E_{\text {Surface }}\right)$ according to a redox reaction of the ferrocene compound immobilized on the surface is derived from the Nernst equation as

$$
E_{\text {Surface }}=E_{\text {Surface }}^{0}-\frac{R T}{F} \ln \left(\frac{[\mathrm{Fc}(\mathrm{II})]_{\text {Surface }}}{[\mathrm{Fc}(\mathrm{III})]_{\text {Surface }}}\right),
$$

where $E_{\text {Surface }}$ is the standard electrode potential, $R$ is the gas constant, $T$ is the absolute temperature, and $F$ is the Faraday constant. The total concentration of the ferrocene compound immobilized on the surface $[\mathrm{Fc}]_{\text {Surface }}$ is expressed as

$$
[\mathrm{Fc}]_{\text {Surface }}=[\mathrm{Fc}(\mathrm{III})]_{\text {Surface }}+[\mathrm{Fc}(\mathrm{II})]_{\text {surface }} \text {. }
$$

In the initial state, almost all ferrocene compounds are in the oxidation state as

$$
[\mathrm{Fc}(\mathrm{III})]_{\text {Surface }} \approx[\mathrm{Fc}]_{\text {Surface. }}
$$

After the redox reaction with the thiol compounds, the concentration of the ferrocene compounds in oxidation state $A$ and the concentration of the ferrocene compounds in reduction state $B$ are shown, respectively, as

$$
\begin{aligned}
& A=[\mathrm{Fc}]_{\text {Surface }}-B, \\
& B=\alpha(\mathrm{R}-\mathrm{SH})_{\text {Liquid }},
\end{aligned}
$$

where $\alpha$ is the reaction efficiency of the redox reaction between the thiol compounds and the ferrocene compounds. Under the condition $[\mathrm{Fc}]_{\text {Surface }} \gg B$, Eq. (4) can be converted using Eqs. (7) and (8), and rewritten as

$$
E_{\text {Surface }}=C-\frac{R T}{F} \ln (\mathrm{R}-\mathrm{SH})_{\text {Liquid }}
$$

where $C$ is constant.

Moreover, under the condition at $25^{\circ} \mathrm{C}$, Eq. (9) can be rewritten as

$$
E_{\text {Surface }}=C-59.2 \log (\mathrm{R}-\mathrm{SH})_{\text {Liquid }} .
$$

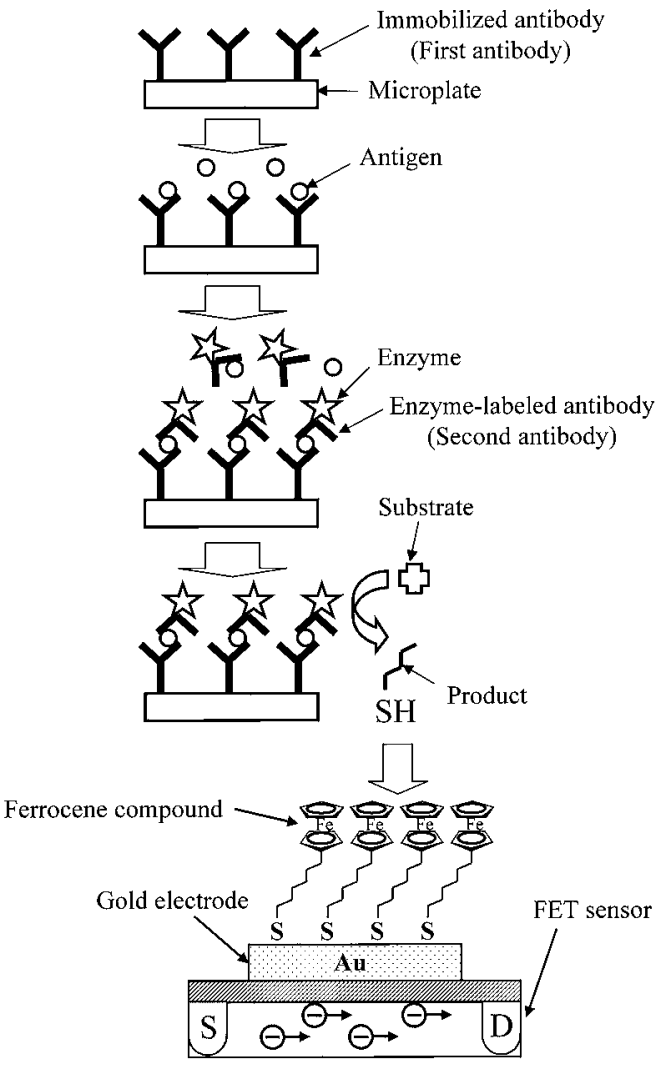

Fig. 7 Principle of enzyme immunoassay by using an extendedgate FET sensor with an 11-FUT modified gold electrode.

Equation (10) shows that a one-order change of the concentration of the thiol compounds will cause an interfacial potential change of the 11-FUT modified gold electrode in 59.2 $\mathrm{mV}$ at $25^{\circ} \mathrm{C}$.

To investigate the redox reaction of the 11-FUT immobilized FET sensor with the thiol compound, a 6-HHT molecule having a SH residue was used as the thiol compound. For normalization, the amount of drain-current shift according to the redox reaction of the 11-FUT immobilized FET sensor was converted into a change in the gate voltage $\left(\Delta V_{\mathrm{g}}\right)$. The $\Delta V_{\mathrm{g}}$ dependency on the concentration of the 6-HHT is plotted in Fig. $6(n=5)$. Here, $\Delta V_{\mathrm{g}}$ shows a fairly good linearity $\left(R^{2}=0.998\right)$ for concentrations from $10^{-6}$ to $10^{-2} \mathrm{M}$ and a Nernstian slope of $58.5 \mathrm{mV} /$ decade for the 6-HHT solution. The concentration of the 6-HHT solution could be obtained promptly and accurately by using an 11-FUT modified FET sensor. In this experiment, since the 11FUT modified FET sensor was used repeatedly after a treatment with a 5\% hydrogen peroxide solution, all measurements could be performed using a single FET-sensor chip.

\section{Enzyme immunoassay using 11-FUT modified FET sensor}

This assay consists of two steps: a sandwich immunoassay and subsequent detection of enzyme-catalyzed thiol compounds by the FET sensor (Fig. 7). The sandwich immunoassay is the same procedure as described previously. ${ }^{16}$ First, an antigen and an enzyme-labeled antibody (second antibody) are sequentially added to a microplate, in which a first antibody is immobilized. After bound/free (B/F) separation, an enzyme substrate solution is added to the microplate, and a thiol compound is produced by the labeled enzyme. Next, the produced thiol compound is determined by measuring the drain-current change of the 


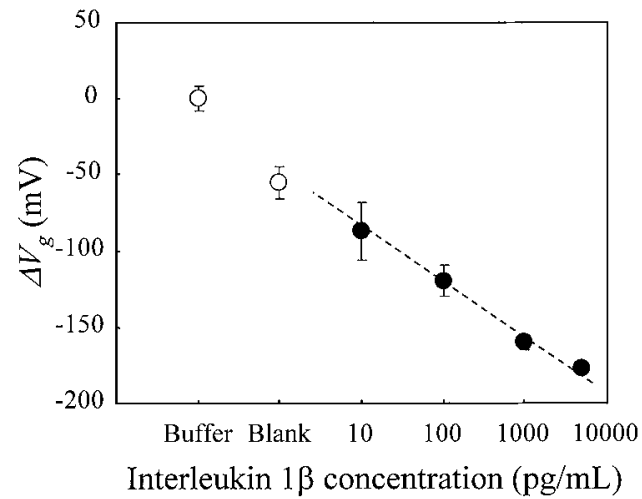

Fig. 8 Concentration of IL-1 $\beta$ versus gate-voltage change $\left(\Delta V_{\mathrm{g}}\right)$ measured by the 11-FUT modified FET sensor.

11-FUT modified FET sensor. Before the thiol compound is introduce onto the gold electrode of the FET sensor, the draincurrent value is constant. After the thiol compound onto the gold electrode is introduced, a redox reaction of the ferrocene compound with the thiol compound occurs on the gold electrode, and the drain current decreases according to the decrease in the interfacial potential on the gold electrode. The amount of thiol compound can be calculated by measuring the drain-current change.

The stability of the interfacial potential change on the gold electrode of the FET sensor was improved by applying a measurement technique using a superimposed high-frequency voltage. ${ }^{16}$ In this study, therefore, an enzyme immunoassay using the 11-FUT modified FET sensor was performed with a $0.1-\mathrm{V}$ DC voltage with a high-frequency sine wave $(1 \mathrm{MHz}, 0.2$ Vp-p) superimposed onto the reference electrode. Though 200 $\mu \mathrm{L}$ of the product solution of the AChE-catalyzed reaction was commonly used in the spectrophotometric method, $10 \mu \mathrm{L}$ of that was used in this study. The dependency of $\Delta V_{\mathrm{g}}$ on the concentration of the IL- $1 \beta$ solution is plotted in Fig. $8(n=3)$. The measured data for a concentration of $0 \mathrm{pg} / \mathrm{mL}$ was used as a blank. And the measured data for only measurement buffer was used as a baseline. For normalization, the amount of the draincurrent shift according to the redox reaction of the 11-FUT immobilized FET sensor was converted into a voltage shift $\left(\Delta V_{\mathrm{g}}\right)$ from the value of the measurement buffer. The value of the $\Delta V_{\mathrm{g}}$ at each concentration was obtained as shown in Fig. 8 . Here, $\Delta V_{\mathrm{g}}$ showed a fairly good linearity $\left(R^{2}=0.995\right)$ with the concentration down to $10 \mathrm{pg} / \mathrm{mL}$ of the IL- $1 \beta$ solution; the detection limit was around $10 \mathrm{pg} / \mathrm{mL}$. This result shows that the detected thiol compound was proportional to the concentration of the antibody-antigen complex (i.e., the concentration of the antigen). A least in principle, since $\Delta V_{\mathrm{g}}$ at a lower concentration is smaller than that at a higher concentration, the fluctuation is expected to be smaller as the case of 6-HHT molecules shown in Fig. 6. Contrary to this expectation, the fluctuation in the measured data at lower concentrations in the enzyme immunoassay became higher than that of higher concentrations, which resulted in a lower sensitivity (detection limit was around $10 \mathrm{pg} / \mathrm{mL}$ ). One major difference between the 6-HHT molecules and the AChE-catalyzed reaction products was impurities in the AChE-catalyzed reaction solution, which would result in large background noise. A further improvement of the detection sensitivity will require removal of the impurities in the AChE-catalyzed reaction solution, or purification of the substrate. In addition, this slope $(37.7 \mathrm{mV} /$ decade $)$ was lower

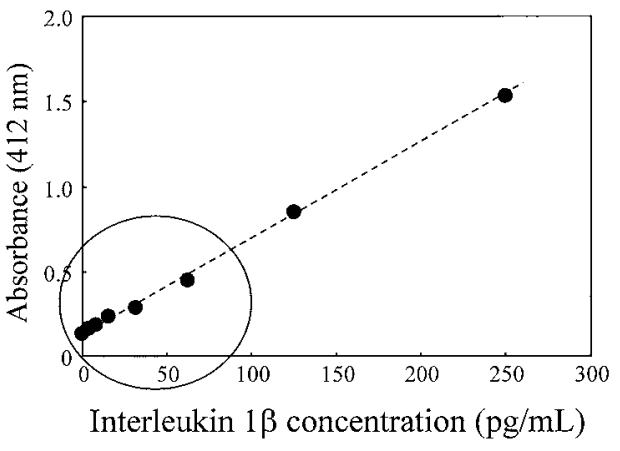

(a)

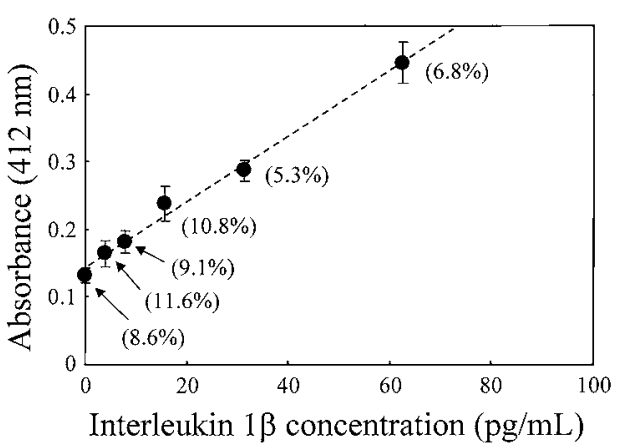

(b)

Fig. 9 Enzyme immunoassay of IL- $1 \beta$ by a common spectrophotometric method using DTNB. (a) Concentration of IL-1 $\beta$ versus absorbance at $412 \mathrm{~nm}$, measured by a spectrophotometric method using DTNB. (b) Enlarged view of curves in the circle area in (a).

than that $(58.5 \mathrm{mV} /$ decade $)$ in the case of the 6-HHT. This difference reflects the difference in the molecular size, and the end group of the thiol compounds, or reflects the lower reproducibility of the manually operated enzyme-immunoassay in this study.

$\mathrm{AChE}$ is well known to be a high-turnover-number enzyme for the hydrolysis of acetylthiocholine. Applying AChE biochemistry to an enzyme immunoassay makes it possible to achieve highly sensitive detection of the substrate. The AChE activity can be determined by a spectrophotometric method using 5,5'-dithiobis-(2-nitrobenzoic acid) (DTNB), which is called "Ellman's reagent". ${ }^{18}$ The immunoassay using DTNB was applied to measure IL- $1 \beta$ at $10 \mathrm{pg} / \mathrm{ml}$ by Grassi et al. ${ }^{19}$ To comparison between the proposed method and the spectrophotometric method using DTNB, the immunoassay for IL- $1 \beta$ was performed by a spectrophotometric method using DTNB in this study. The absorbance was measured at $412 \mathrm{~nm}$, and the detection volume was $200 \mu \mathrm{L}$. The result obtained using the spectrophotometric method using DTNB is shown in Fig. 9(a). An enlarged view of the curves in circle area in Fig. 9(a) is shown in Fig. 9(b). Though the fluctuation (C.V., around 2\%) of the measured data at more than $100 \mathrm{pg} / \mathrm{mL}$ was small, it (C.V., 9 - $12 \%$ ) became higher at less than $20 \mathrm{pg} / \mathrm{mL}$. There was the same tendency in the enzyme immunoassay using the 11-FUT modified FET sensor. The detection limit of the spectrophotometric method using DTNB was ca. $10 \mathrm{pg} / \mathrm{mL}$, which was almost the same value as that in the enzyme immunoassay using the 11-FUT modified FET sensor. Another approach was an enzyme immunoassay combining the AChE biochemistry with electrochemistry, that is, a linear sweep 
voltammetric measurement of B-type natriuretic peptide at 10 $\mathrm{pg} / \mathrm{ml},{ }^{20}$ and a potentiometric detection of IL- $1 \beta$ at $1 \mathrm{pg} / \mathrm{mL}$ by an extended-gate FET sensor. ${ }^{16}$ Though the potentiometric detection method by the extended-gate FET sensor was realized with highly sensitive detection, the sensor could not be reused without any special treatment to remove the adsorbed molecules. Therefore, the sensor tip as well as the reagents must be disposed of. On the contrary, the 11-FUT modified FET sensor can be used repeatedly after a treatment with a $5 \%$ hydrogen peroxide solution. Therefore, the enzyme immunoassay using a redox reaction of 11-FUT immobilized on a gold surface of an extended-gate FET sensor can overcome this disadvantage. In the future, to take advantage of the reusable sensor, enzyme immunoassay using the 11-FUT immobilized FET sensor will be applied to other targets not requiring a high sensitivity of less than $10 \mathrm{pg} / \mathrm{mL}$. In addition, since the sensitivity of potentiometric detection does not depend on the detection volume, the FET senor can detect a small volume without reducing the sensitivity. In this study, it detected a small sample volume of less than $10 \mu \mathrm{L}$. If a small sample volume of less than $10 \mu \mathrm{L}$ can be prepared, a smaller ELISA system will be realized and can be applied for in clinical-medicine and environmental applications.

\section{Acknowledgements}

We would like to thank Dr. T. Anazawa and Mr. T. Matsunaga (Central Research Laboratory, Hitachi, Ltd., Japan) for their assistance and useful discussions.

\section{References}

1. A. Warsinke, A. Benkert, and F. W. Scheller, Fresenius J. Anal. Chem., 2000, 366, 622.

2. M. Díaz-González, M. B. González-García, and A. CostaGarcía, Electroanalysis, 2005, 17, 1901.
3. J. S. Rossier and H. H. Girault, Lab Chip, 2001, 1, 153.

4. J. Das, M. A. Aziz, and H. Yang, J. Am. Chem. Soc., 2006, 128,16022

5. J. Das, K. Jo, J. W. Lee, and H. Yang, Anal. Chem., 2007, 79,2790

6. J. F. Schenck, "Theory, Design and Biomedical Application of Solid State Chemical Sensors", 1978, ed. P. W. Cheung, CRC Press, Boca Raton, FL.

7. C.-L. Feng, Y.-H, Xu, and L.-M. Song, Sens. Actuators, B, 2000, 66, 190.

8. R. B. M. Schasfoort, P. Bergveld, R. P. H. Kooyman, and J. Greve, Anal. Chim. Acta, 1990, 238, 323.

9. R. B. M. Schasfoort, R. P. H. Kooyman, P. Bergveld, and J. Greve, Biosens. Bioelectron., 1990, 5, 103.

10. J. C. Van Kerhof, J. C. T. Eijkel, and P. Bergveld, Sens. Actuators, B, 1994, 18, 56.

11. J. C. T. Eijkel, W. Olthuis, and P. Bergveld, Biosens. Bioelectron., 1997, 12.991.

12. G. A. J. Besselink, R. B. M. Scasfoort, and P. Bergveld, Biosens. Bioelectron., 2003, 18, 1109.

13. W. E. Lee, H. G. Thompson, J. G. Hall, and D. E. Bader, Biosens. Bioelectron., 2000, 14, 795.

14. Z. E. Selvanayagam, P. Neuzil, P. Gopalakrishnakone, U. Sridhar, M. Singh, and L. C. Ho, Biosens. Bioelectron., 2002, 17, 821.

15. Y. V. Plekhanova, A. N. Reshetilov, E. V. Yazynina, A. V. Zherdev, and B. B. Dzantiev, Biosens. Bioelectron., 2003 , 19, 109.

16. M. Kamahori, Y. Ishige, and M. Shimoda, Biosens. Bioelectron., 2007, 22, 3080.

17. Y. Ishige, M. Shimoda, and M. Kamahori, Jpn. J. Appl. Phys., 2006, 45, 3776.

18. G. L. Ellman, Arch. Biochem. Biophys., 1959, 82, 70.

19. J. Grassi, Y. Frobert, P. Pradelles, F. Chercuitte, D. Gruaz, J.-M. Dayer, and P. E. Poubelle, J. Immunol. Methods, 1989, 123, 193.

20. H. Matsuura, Y. Sato, O. Niwa, and F. Mizutami, Anal. Chem., 2005, 77, 4235. 